\title{
Ficha analítica para el estudio morfo-tecnológico de la industria lítica no reelaborada
}

\author{
Francisco Javier Muñoz Ibáñez */** \\ Juan Rodríguez de Tembleque Moreno ** \\ José Ramón López Moreno de Redrojo **
}

\section{INTRODUCCIÓN}

En los últimos años han venido apareciendo diferentes propuestas metodológicas para el estudio de la industria lítica no reelaborada. Así, se ha ido poniendo de manifiesto la importancia del examen de este tipo de materiales que, frecuentemente, eran ignorados o se procedia a un simple recuento numérico. La aparición de trabajos como los de Bemaldo de Quirós et al., (1981); Carbonell, E., et al. (1983b); Baena, J., y Luque, M. (1990); o Inizan, M.L., et al. (1992); han venido a rellenar el vacío metodológico existente. La ficha de trabajo que presentamos trata de aunar las propuestas antes citadas y ampliar la información que nos proporciona este tipo de material.

Para su realización se ha tenido en cuenta la especificidad de la industria objeto de estudio, material de superficie variado en lo que a materia prima, alteraciones e, incluso, adscripción cultural respecta, el cual procede de las prospecciones llevadas a cabo en el yacimiento Pedazo del Muerto (Pinto, Madrid). Estos conjuntos industriales suelen ser muy problemáticos por el contexto en que aparecen; no obstante su importancia intrínseca hace necesario un tratamiento metodológico que nos permita obtener la máxima información posible.

* Becario de FPI de la UNED.

* Coordinadores del "Proyecto de Prospección y Estudio del yacimiento Pedazo del Muerto (Pinto, Madrid)" y colaboradores en diferentes proyectos de investigación. 
También ha influido en su confección la metodologia ${ }^{1}$ y los objetivos planteados ${ }^{2}$, así como el programa informático ${ }^{3}$ empleado como soporte de la misma. Todo ello nos ha llevado al desarrollo de una ficha exhaustiva desde el punto de vista descriptivo, pero que aporta información muy útil para la caracterización y estudio estadistico del material lítico, pudiéndose aplicar con independencia que presente retoque o no.

La ficha elaborada, aunque esté especialmente diseñada para este tipo de yacimiento, podrá ser aplicada a diferentes problemáticas particulares con pequeñas modificaciones o eliminando aquellos campos que no sean de utilidad, bien por el dato en sí mismo o porque ya se conozca su evolución.

\section{DESCRIPCIÓN GENERAL}

La ficha consta de varios bloques conceptuales que se disponen horizontalmente. Los encabezamientos siguen una lógica desde lo más elemental, general o natural (referencias de control, descripción de las materias primas, alteraciones de dichas materias primas...), a lo más concreto, complejo y antrópico (magnitudes y atributos tecnológicos, p.e.). En su desarrollo vertical, cada bloque consta de una serie de apartados y de subapartados que a su vez pueden dividirse en función de las necesidades descriptivas o de nuestros intereses, y siempre que sea posible

Metodología: Prospección sistemática intensiva periódica.

2 Objetivos: mo.

a) Estudio a nivel de superficie del yacimiento y su relación con el entorno próxi-

b) Viabilidad, potencialidad informativa y rentabilidad de la prospección microespacial para dicho estudio y el conocimiento de la incidencia antrópica y medio ambiental en los restos arqueológicos.

c) Valoración de este tipo de estudios en sí mismos y bajo una perspectiva espacial más amplia.

3 Programa informático: Excel 4. 
y oportuno, en cuatro subapartados finales, según se expresa en el diagrama siguiente:

\section{CONCEPTO GENERAL}

\section{CARACTERÍSTICA PARTICULAR}

$\begin{array}{cccc}\text { GRADO } & \text { ZONA AFECTADA } & \text { ORIGEN } & \text { CLASE } \\ \text { O } & O & O & 0 \\ \text { INTENSIDAD } & \text { LOCALIZACIÓN } & \text { CAUSA } & \text { TIPO }\end{array}$

También se han introducido campos temporales, como la cronología de la fracturas y de los pseudorretoques para, de esta forma, especificar si son antiguos o recientes.

A la hora de describir determinadas características se ha optado por simplificar los campos, reduciendo éstos a la presencia o no de la misma y al grado o influencia zonal general en la pieza, debido fundamentalmente al desconocimiento que sobre las mismas se tiene actualmente 0 a la escasa información que nos aporta.

A continuación, se explica cada uno de los cuatro subapartados finales.

Grado.- Intensidad con que se manifiesta una característica. Puede ser cuantificable y expresarse mediante números $(1,2,3 \ldots) \circ$ intervalos numéricos (p.e., $30<\%<60$ ). Cuando no es posible asignar una cantidad a una cualidad, empleamos cuatro gradaciones subjetivas de intensidad que denominamos de menor a mayor o de menos a más:

MB (Muy Bajo) $\rightarrow$ Estadio núm. $1 \rightarrow$ Leve, incipiente, muy poco/s. $\mathrm{B}$ (Bajo) $\rightarrow$ Estadio núm. $2 \rightarrow \mathrm{Poco} / \mathrm{s}$, algunos, significativo. $M$ (Medio) $\rightarrow$ Estadio núm. $3 \rightarrow$ Bastante/s, grado entre estadios 2 y 3. A (Avanzado) $\rightarrow$ Estadio núm. $4 \rightarrow$ Avanzado, intenso, muchos.

Zona afectada.-Expresa el lugar de la pieza donde se localiza o manifiesta la característica que se está describiendo. A veces, indirectamente, también expresa intensidad ("cuánto") y modo ("cómo"). Así, por ejemplo, cuando decimos que una pieza está desilicificada parcialmente, estamos expresando a la vez un concepto zonal y un grado de alteración superficial. Sin embargo, una "cualidad" puede afectar localmente a la pieza, pero de manera muy intensa. Por otra parte, cuando hablamos, por ejemplo, de pseudorretoques situados en la zona mesial de forma continua, estamos también haciendo referencia a cómo se dis- 
tribuyen o se presentan dichos pseudorretoques en una zona concreta. En todo caso, se han usado los siguientes términos zonales:

$\begin{array}{llll}\text { Local } & \text { Anverso } & \text { Proximal } & \text { Aislados } \\ \text { Parcial } & \text { Reverso } & \text { Mesial } & \text { Próximos } \\ \text { General } & \text { Talón } & \text { Distal } & \text { Contiguos } \\ & \text { Canto } & & \end{array}$

Causa.-Supuesto origen de la caracteristica que estamos describiendo. Es una apreciación bastante subjetiva, pero que puede resultar útil para los estudios que sobre temas concretos están llevando a cabo algunos investigadores. Así, por ejemplo, no siempre es fácil discernir cuando la rebaba que presenta el extremo distal de un soporte ha sido causada por una reflexión (en definitiva, una flexión pasiva) o una fractura distal por flexión. Muchas fracturas presentan planos lisos, incluso rectos, sin huellas de percusión (negativos de bulbos, p.e.) cuyo origen se desconoce. Sin embargo, en otros casos sí existen criterios que nos inducen a pensar, con las debidas reservas, en una atribución probable (véase estudio de fracturas y pseudorretoques). Análogamente a lo dicho anteriormente para las localizaciones, cuando hablamos de las causas que originen una determinada característica, indirectamente estamos definiendo tipos de la misma (pseudorretoques de uso, fracturas por flexión, etc.).

Tipos.-Modelo ideal que reúne todos los parámetros por los que se define (p.e., tipos de materia prima, soportes y accidentes de talla).

Se ha procurado objetivar el mayor número de características, haciéndolas cuantificables (magnitudes, presencia/ausencia, elementos contables...). Sin embargo, en otras como el color, la textura, el comportamiento ante la luz, el grado de rodamiento o la intensidad de algunos de sus atributos, no ha sido posible, a simple vista, cuantificarlas o establecer límites precisos, por lo que en última instancia su valoración viene determinada por la apreciación subjetiva del investigador. Para paliar, en la medida de lo posible, este problema conviene que el equipo de trabajo utilice criterios uniformes en sus apreciaciones, por lo que se recomienda el análisis de las primeras unidades en grupo para definir muestras-patrones de referencia.

\section{BLOQUE I $\rightarrow$ REFERENCIA (Véase gráfico 1)}

Como en toda ficha, lo primero que se ha introducido son los datos de referencia o control de cada unidad lítica que queremos describir:

Datos relativos al control de la pieza:

NUM $\rightarrow$ Referencia numérica asociada a cada pieza. 
DIB $\rightarrow$ Dibujo. Este campo se rellena cuando la pieza merece la pena ser dibujada, bien porque esté retocada, sea un tipo característico, o cualquier otro motivo de interés para el investigador.

FOT $\rightarrow$ Fotografía. Del mismo modo al caso anterior, se rellenará siempre que resulte de interés fotografiar la pieza. En la casilla correspondiente se pondrá un número correlativo de referencia, seguido de un asterisco cuando la pieza sea fotografiada formando parte de un grupo.

Datos relativos a la prospección (PROSP):

$\mathbf{A N} \rightarrow$ Año en que fue recogida la muestra.

AR $\rightarrow$ Area, subdivisión general de la zona prospectada intensivamente. Como en nuestro caso, pueden utilizarse las iniciales de los topónimos que figuran en los mapas 1:5.000 (p.e., PM=Pedazo del Muerto).

SE $\rightarrow$ Sector, subdivisión de un área. Para su denominación se han empleado letras $(A, B, C . .$.$) . A su vez, éstos pueden dividirse en subsecto-$ res, para cuya denominación hemos añadido a las letras correspondientes al sector que pertenecen un subíndice numérico $\left(A_{1}, A_{2}, \ldots, B_{1}, B_{2}, \ldots\right.$, etc.). Esta información deberá figurar en la casilla cuando se disponga de ella, ya que, implícitamente, conoceremos el sector y el área a que pertenece. En los casos que no interese o que, por cualquier razón, no se pueda precisar a qué subsector o sector pertenece la pieza, se pondrá en la casilla las referencias de los dos sectores o subsectores adyacentes (p.e., J-E).

Cuando se trate de "material procedente de una excavación" deberán figurar en este apartado los datos relativos a la cuadricula, estrato, nivel, etc.

\section{BLOQUE II $\rightarrow$ MATERIA PRIMA (Néase gráfico 1)}

En este bloque se intenta individualizar los diferentes tipos y subtipos de materia prima utilizados y ver qué caracteristicas los definen, su rareza o abundancia; para a partir de aquí, intentar localizar su proce-

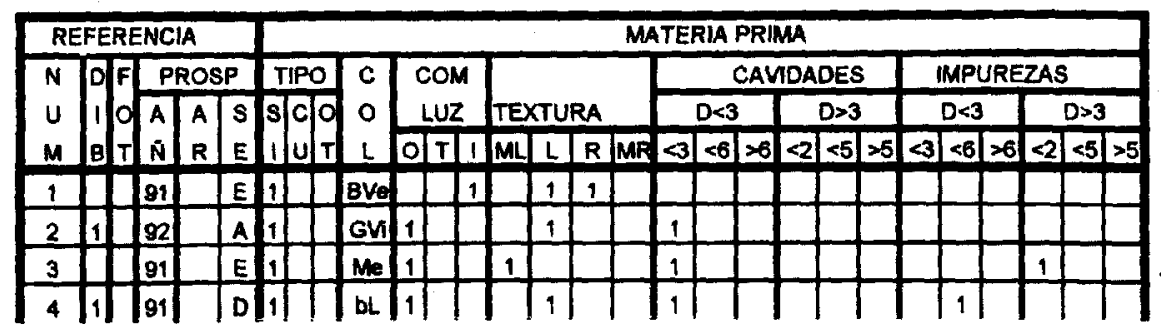

Gráfico 1: Bloques | y |l. 
dencia y poder conocer o al menos intuir su facilidad de obtención, tamaño, posibilidad de transporte, etc.

Asimismo, y debido al predominio de diferentes "variedades" de sílex, hemos considerado oportuno desarrollar criterios descriptivos como color, comportamiento ante la luz, o textura, que posibiliten su identificación. Estos criterios, un tanto subjetivos en cuanto a su apreciación, pueden ser válidos o útiles para cada yacimiento concreto siempre que se clarifiquen y expresen netamente las características de las muestras de cotejo o referencia.

Como criterios objetivos se contemplan la presencia (y la cantidad) o ausencia de oquedades e impurezas que pueden facilitar la identificación de materias primas procedentes de un mismo nódulo, plaqueta o núcleo - si bien éstos pueden tener una estructura heterogénea- o de una misma unidad geológica. Estas variables, junto con alguna del siguiente bloque, pueden orientarnos a la hora de estudiar la posible existencia de remontajes o las distintas calidades de los sílex empleados, y las consecuencias que de estos estudios puedan derivarse (p.e., aptitud para la talla), además de ayudar a la comprensión de la dinámica de formación del yacimiento.

Tipo.-Nos referimos a las diferentes clases de rocas que se presentan en el yacimiento. Se ha considerado, en principio, sólo tres tipos de materia prima, ya que en el yacimiento objeto de estudio hemos recogido básicamente diferentes variedades de silex, y muy raramente cuarcita y otras (p.e., caliza).

$$
\begin{aligned}
& \text { SI } \rightarrow \text { Silex. } \\
& \text { CU } \rightarrow \text { Cuarcita. } \\
& \text { OT } \rightarrow \text { Otras rocas. En nuestro caso caliza, cuarzo... }
\end{aligned}
$$

Col.-Color dominante. Se expresa mediante un código formado por una o más letras mayúsculas y/o minúsculas (p.e., G-Vi=Gris Violáceo).

Com Luz.-Comportamiento ante la luz. Capacidad de absorción de la luz por la materia prima. En relación con los componentes minerales, sus estructuras y texturas petrológicas, impurezas de la roca, moléculas de agua que contiene.

$0 \rightarrow$ Opaco. Cuando la materia prima absorbe totalmente la luz.

$\mathbf{T} \rightarrow$ Traslúcido. Cuando a través de la materia prima pasa una parte significativa de la luz incidente.

I $\rightarrow$ Caso intermedio entre los dos anteriores.

La observación de la opacidad o transparencia de la materia prima se realizará sobre las partes más delgadas de la pieza, utilizando siempre las mismas o semejantes condiciones de exposición a la luz. Debe 
tenerse en cuenta que, a igualdad de materia prima, cuanto más espesa sea la pieza más luz absorberá. A veces una misma pieza puede ser parcialmente opaca y traslúcida por ser la materia prima heterogénea.

Textura.-Granulometría de los elementos superficiales que componen una roca y su sensación al tacto. Puede ocurrir que, por las mismas circunstancias que el caso anterior, una pieza presente dos o más texturas. En dicho caso, se rellenarán las casillas correspondientes al grado de cada textura.

$$
\begin{aligned}
& \text { ML } \rightarrow \text { Muy lisa. } \\
& \mathbf{L} \rightarrow \text { Lisa. } \\
& \mathbf{R} \rightarrow \text { Rugosa. } \\
& \text { MR } \rightarrow \text { Muy rugosa. }
\end{aligned}
$$

Cavidades.-Oquedades, por lo general, longitudinales (surcos) o de tendencia circular que presenta la superficie de la pieza. Separamos en sendos subapartados aquellas cuya longitud máxima (D) es menor de 3 $\mathrm{mm}$., de las que tienen dicha magnitud mayor, y las contabilizamos a intervalos: $<3,<6$ y $>6$, para $\mathrm{D}<3 \mathrm{~mm}$., y $<2,<5$ y $>5$, para $\mathrm{D}>3 \mathrm{~mm}$.

Impurezas.-Intrusiones de la roca-caja que son perceptibles en la superficie de la pieza. A veces puede confundirse con residuos de córtex. Pueden presentar formas variadas, pero por lo general son pseudocirculares. Para contabilizarlas se ha empleado el mismo esquema anterior.

\section{BLOQUE III $\rightarrow$ ALTERACIONES DE LA MATERIA PRIMA (Véase gráfico 2)}

«Para profundizar en el concepto de industria, es esencial distinguir entre lo natural, lo accidental y lo intencional" (Inizan, M.L., et al., 1992, pág. 27). En este sentido, la observación metódica y detenida de la superficie de un objeto lítico es fundamental. Las diferentes alteraciones que

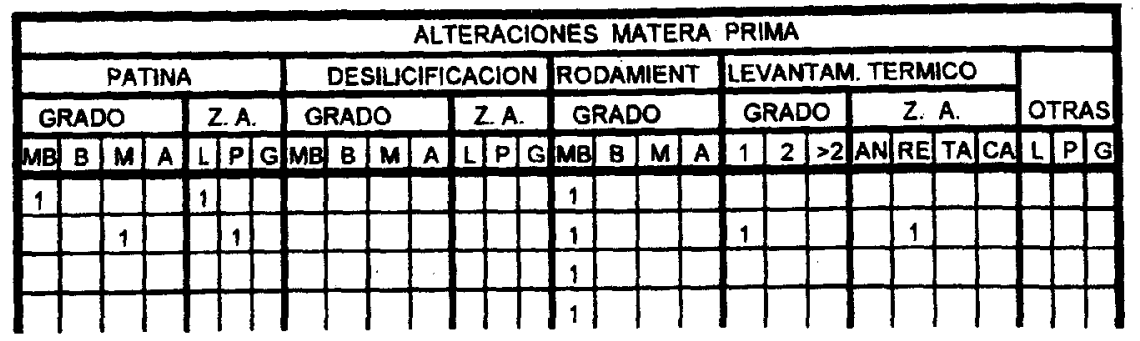

Gráfico 2: Bloque III. 
presenten estas superficies pueden ayudar a aclarar el carácter de las mismas. Igualmente, pueden aportarnos información sobre el tratamiento y reutilización de la materia prima, los procesos postsedimentarios, la "historia" del artefacto hasta su recuperación, etc.

Sin embargo, si la propia génesis del silex es un fenómeno complejo sobre el que no hay unanimidad entre los especialistas en rocas siliceas, toda incursión de los arqueólogos en el campo de algunas de las alteraciones de los sílex es banal, aunque sea meramente descriptiva, si no aporta nada al conocimiento tecnológico y cultural del horizonte que nos ocupa, y nada nos explica sobre la formación del yacimiento, los procesos que le han afectado y su cronología. Téngase en cuenta, además, que muchos de los procesos de alteración del sílex pueden ser anteriores a la manipulación de éstos por parte del hombre, complicando aún más cualquier conclusión positiva.

Por otra parte, algunos tipos de rocas, como las metamórficas, son, por lo general, más resistentes a determinadas alteraciones, o ésas son menos perceptibles. Incluso dentro de las propias rocas siliceas, también existen diferentes grados de respuesta ante los mismos fenómenos.

Pátina.-Toda alteración de la superficie de la pieza que determina un cambio en su respuesta ante la luz, y que en ocasiones le confiere incluso una cierta coloración (p.e., tonalidades blanquecinas o azuladas) y textura diferente a las originarias. Existen múltiples factores físicos y/o químicos que pueden producir alteraciones de este tipo. Así, una pieza expuesta al fuego o simplemente a la intemperie, sometida a acciones mecánicas naturales o de uso, o afectada por procesos químicos que tienen lugar en el medio edafológico, etc., puede cambiar el aspecto de su superficie en los términos antes expuestos. Podemos, pues, afirmar que es prácticamente imposible que llegue hasta nosotros una pieza lítica prehistórica sin que presente pátina. Lo interesante, por tanto, sería conocer su origen, pero éste puede ser múltiple. Nos queda entonces la única opción de especificar los casos en que la pieza presente dos tipos de pátinas, bien porque haya sido reutilizada con posterioridad, presente facturas o desconchados recientes o una de sus partes haya sufrido un proceso físico y/o químico diferente del resto de la pieza (por uso, fuego, contacto con sales minerales, etc.).

Por otra parte, la cuestión se complica al no ser por lo general la materia prima homogénea, presentando su superficie diferente resistencia a estos fenómenos. Así pues, hablaremos de pátina sólo cuando una parte de la superficie presente un brillo o tonalidad diferenciada.

Desilicificación.-Algo parecido ocurre con la alteración denominada por unos desilificación, por otros desilicificación, y antaño, deshidra- 
tación. Se trata de un proceso todavía no bien comprendido mediante el cual, al parecer por disolución, la materia pierde sílice, y con ella resistencia mecánica, elasticidad y peso, a la vez que adquiere un color blanquecino y un aspecto tizoso, que puede llevarnos a confusión con los restos o residuos de la roca-caja cuando ésta es caliza o entre sus minerales se encuentra la calcita.

Para la pátina y la desilicificación hemos considerado los cuatro estadios de grado: MB, B, M y A; y tres campos zonales (Z.A.): L=Local, $\mathbf{P}=$ Parcial, $\mathbf{G}=$ General; en función de si la alteración ha afectado solamente a una porción pequeña de la pieza, a una parte, $o$ a toda la pieza, respectivamente.

Rodamiento.-Carácter o aspecto romo que adquieren vértices, filos y aristas como consecuencia de procesos mecánicos y, más raramente, químicos. Por lo general, el rodamiento altera a toda la pieza de una manera homogénea, redondeando por igual vértices, filos y aristas, por lo que hemos prescindido de los campos zonales y sólo hemos tenido en cuenta el grado (MB, B, M y A) con que se presenta tal alteración. No obstante, pueden existir piezas talladas sobre materia prima rodada, o piezas rodadas y posteriormente reutilizadas que presenten dos grados diferentes de rodamiento según zonas. Otras veces la pieza está constituida' por materia prima heterogénea, por lo que el rodamiento puede afectar de manera diferente a sus partes. Algunos autores prefieren hablar de "redondeamiento" para no prejuzgar el origen de la alteración, ya que ésta puede tener otras causas, como por ejemplo el embotamiento por uso; pero en estos casos dicha alteración suele ser somera y afectar tan sólo a una parte de la pieza.

Levantam. Térmico.-Levantamientos térmicos. Negativos de extracciones no antrópicas consecuencia de cambios bruscos de temperatura o de temperaturas extremas, que presentan una superficie marcadamente cóncava y sin ninguna huella de presión o percusión. En la ficha se especifica si la pieza tiene 1, 2 ó >2 levantamientos (grado). Su localización exacta no aporta información relevante al prehistoriador, por lo que solamente se expresa la zona afectada (Z.A.) de forma general: anverso (AN), reverso (RE), talón (TA) o canto (CA).

Otras.-En este apartado contemplamos otras alteraciones térmicas (cuarteado: C, craquelado: CR y rubefacción: $\mathbf{R})^{4}$ que debido a su esca-

4 Cuarteado: Aspecto reticulado que adquiere la superficie de la lasca tras estar expuesta a temperaturas extremas o cambios bruscos de temperatura. 
sa o nula presencia en el yacimiento en estudio no las hemos considerado de forma individualizada. Sólo las constatamos mediante su letra de identificación en los campos L/P/G, pues no nos interesa precisar más.

\section{BLOQUE IV $\rightarrow$ PSEUDORRETOQUES (Néanse gráficos 3a y 3b)}

Extracciones no intencionadas, generalmente de pequeño tamaño, consecuencia de procesos naturales 0 antrópicos.

Debido a la naturaleza de la industria en estudio, en posición secundaria, y por el alto porcentaje de pseudorretoques que presenta, hemos optado por darle un tratamiento detallado.

Grado.-En primer lugar, se ha considerado oportuno evaluar de forma global el grado de alteración de la pieza, estableciendo intervalos de porcentajes de filo pseudorretocado respecto al total de filo que presenta la misma. Para definir dichos intervalos hemos empleado 30,60 , 90,100 , expresando cada cifra el intervalo de porcentaje de filo afectado que va desde la anterior cifra hasta la que ella determina, salvo en el caso de 30, que hace referencia a un tanto por ciento comprendido entre cero y dicha cantidad. Cuando no se observen pseudorretoques, no se rellenará ninguna casilla.

Lado derecho/lado izquierdo.-Un estudio más pormenorizado se realiza posicionando (localización) los pseudorretoques longitudinalmente en los filos laterales de la pieza, empezando por el anverso y continuando con el reverso. De esta forma analizaremos, para anverso (AN) y reverso (RE), si cada filo lateral presenta pseudorretoques en su zona proximal (P), zona mesial (M), y/o zona distal (D), y si aparecen aislados (A), próximos (P), y/o contiguos (C).

A continuación, se tratará de asignar una causa a los mismos, especificando si su origen pudo ser debido al uso (US), transporte (TR) o causa indeterminada (IN).

La uniformidad o no de los pseudorretoques, los tipos (morfología y tamaño), la posición que ocupan en la pieza, su distribución, y la pre-

Craquelado: Carácter irregular y rugoso de la superficie de la lasca producido al saltar de aquélla bruscamente múltiples fragmentos o esquirlas como consecuencia de las tensiones que soporta por la acción del fuego o del frío intenso.

Rubefacción: Cambio en la tonalidad del color y del brillo de la materia prima al experimentar un calentamiento prolongado o intenso. 
Ficha analítica para el estudio morfo-tecnológico de la industria lítica no reelaborada

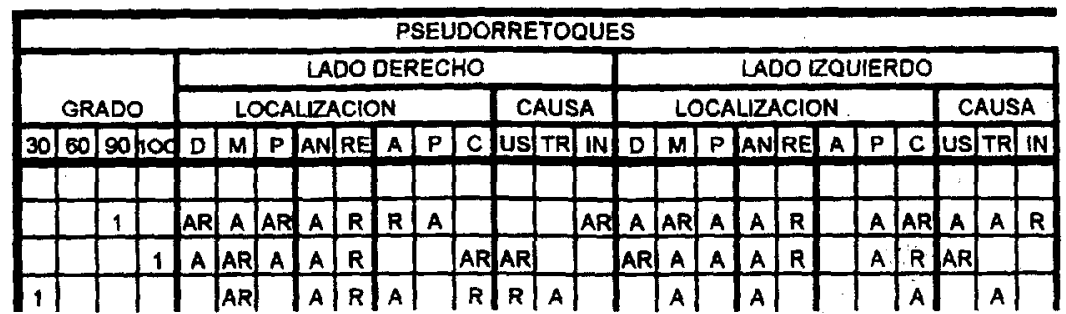

Gráfico 3a: Bloque IV.

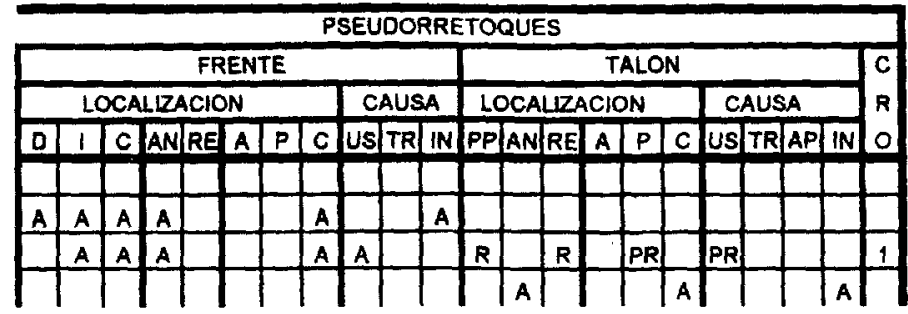

Gráfico 3b: Bloques IV.

sencia o ausencia de pseudorretoques en otras partes de la pieza -incluyendo las aristas- son criterios que han de tenerse en cuenta a la hora de pronunciarnos sobre su origen. Por lo general, hemos atribuido al uso pseudorretoques continuos y más o menos uniformes situados en una de las caras, y cuya cara opuesta del mismo filo no presenta pseudorretoques, o si los tiene son escasos y aislados, a veces próximos. Aquellos pseudorretoques aislados, heterogéneos en cuanto a tamaño y forma, que se presentan aleatoriamente a lo largo de un filo o de gran parte de la pieza, los hemos asociado al transporte, incluyendo en este concepto las alteraciones producidas por impactos o presiones de todo tipo que ha sufrido la pieza.

Las casillas AN (anverso) y RE (reverso) se rellenarán con una " $A$ " y una " $R$ », respectivamente, cuando el filo correspondiente presente pseudorretoques en dichas caras. El resto de las casillas de localización y causa se rellenarán con una " $A$ ", con una "R" o con " $A R$ ", según se cuentren los pseudorretoques en el anverso, reverso o en ambas caras, respectivamente.

Frente.-Además de los filos laterales, algunas piezas presentan filo transversal al eje tecnológico, que hemos denominado frente, y que ana- 
lizamos de manera similar a los filos laterales de la pieza, aunque en este caso, al estar situado el filo en el extremo distal, la localización de los retoques se estudia en relación a la posición que ocupan respecto al citado eje. Para ello hemos dividido el frente en tres tramos: el derecho (D), el izquierdo (I) y el central (C). Esta subdivisión adquiere mayor o menor relevancia según la longitud del frente.

Por lo que respecta a la causa se seguirá el mismo criterio metodológico que en el apartado anterior.

Talón.-También el talón puede presentar pseudorretoques, muy a menudo en la interseccion con el anverso - probablemente en relación con la eliminación de la cornisa o la preparación del punto de impacto$y$, menos frecuentemente, en la confluencia con el reverso. Estos pseudorretoques, pues, afectan tanto a anverso/reverso como al «plano de percusión" (PP), ya que todo pseudorretoque en el extremo de la superficie del talón "come" extremo proximal de anverso o reverso, y viceversa, por lo que no tiene sentido estudiar éstos aisladamente (en realidad se trata de los mismos pseudorretoques).

No obstante, pondremos una " $A$ » o una " $R$ " en las casillas correspondientes a anverso o reverso cuando los pseudorretoques arranquen del talón pero se extiendan por dichas caras, respectivamente. Cuando los pseudorretoques partan de anverso y/o reverso pero invadan la superficie del talón, rellenaremos con una " $P$ " la casilla correspondiente al "plano de percusión", y lo mismo haremos en la casilla asignada a la cara de la lasca de la que parten.

De esta forma, lo que en realidad se constata es la dirección dominante de los pseudorretoques: de tendencia ortogonal a la superficie del talón $(" A » y$ « $R »)$, cuando los impactos o presiones han tenido lugar preferentemente sobre dicha superficie, extrayéndose más porción de anverso (A) o de reverso (R) que de talón; y de tendencia rasante a la misma superficie, cuando las acciones que las ocasionaron incidieron en general perpendicularmente al anverso $o$ al reverso, desprendiéndose en este caso más superficie de talón que de una de las dos caras de la pieza.

También en este caso, la causa se estudiará de forma análoga al primer apartado de este bloque. Además, se ha introducido un nuevo campo (AP) que contempla los pseudorretoques originados en el acondicionamiento del plano de percusión o presión (abatimiento de cornisa, preparación del punto de impacto, ...).

En ocasiones las "aristas" puden presentar pseudorretoque, circunstancia que se recogerá en el apartado de observaciones o, si el fenómeno es frecuente, puede ser rentable abrir en la ficha un nuevo campo para tal fin. 
CRO.-Cronologia. Algunos de los pseudorretoques de "transporte" pueden deberse a la acción antrópica reciente, en cuyo caso suelen aparecer, aleatoriamente, desconchados aislados y frescos (con diferente pátina) de cierto tamaño, que a veces forman muescas, así como uretoques" muy abruptos que "muerden" el filo y que raramente conforman filos denticulados. Por tal motivo, este apartado se rellenará cuando los pseudorretoques de "transporte" sean recientes.

\section{BLOQUE $V \rightarrow$ FRACTURAS (Néase gráfico 4)}

Decimos que una pieza está fracturada cuando al menos ha perdido una parte del contorno de la misma, presentando una superficie de rotura que afecta a su tipometría. Las fracturas pueden ser intencionadas o no, y producirse por diferentes causas.

Debido a la procedencia superficial de la muestra, a los objetivos planteados y al creciente interés por este tipo de atributo o accidente, hemos desarrollado este apartado al objeto de contabilizar su número, zonas de la pieza afectadas, morfologias de las mismas (delineación longitudinal y transversal y atributos), y, en función de éstas, la posible causa. También señalamos si las fracturas son recientes.

Número.-Se contabiliza si el soporte tiene 1, 2, $30>3$ fracturas.

Zona afect.-Zona afectada. Para describirla manejamos los términos proximal (PR), distal (DI), lateral izquierdo (LI) y lateral derecho (LD). Los dos primeros se pueden combinar con los dos últimos, indistintamente, cuando las fracturas afecten a partes significativas de un extremo y de un lateral, es decir, se presenten oblicuas respecto al eje tecnológico de la pieza. En estos casos se rellenarán las dos casillas correspondientes. De esta forma, las posibilidades que pueden darse, empezando por la zona distal y siguiendo el sentido de las agujas del reloj,

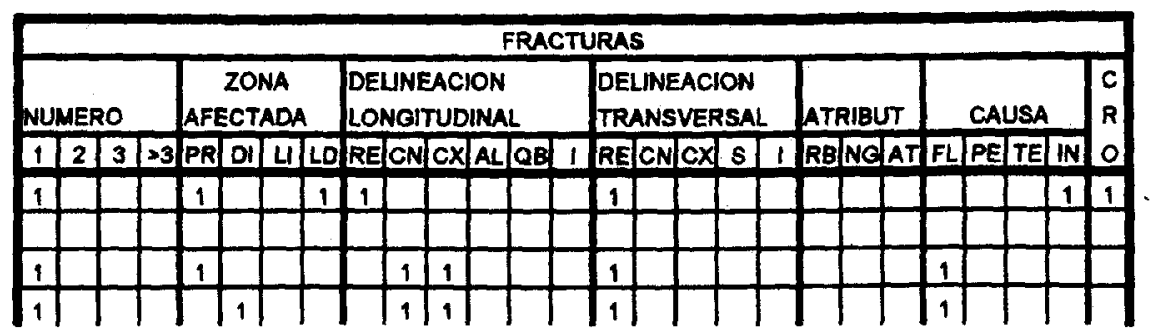

Gráfico 4: Bloque V. 
son: D, D-LD, LD, LD-P, P, P-LI y LI-D. Cuando la pieza tenga más de una fractura, al rellenar las casillas se pondrán signos distintivos de cada una de ellas, para así poderlas situar mentalmente o hacer estudios estadísticos.

Delineación Iongitudinal.-Entendemos por tal la forma predominante de las líneas de intersección del plano de la superficie de fractura con cada uno de los planos paralelos al plano de lascado, pudiendo adoptar una forma recta (RE), cóncava (CN), convexa (CX), sinuosa (SI), quebrada (QB), o irregular (I).

Delineación transversal.-Análogamente al caso anterior, forma predominante de las líneas resultantes de la intersección del plano de la superficie de fractura con cada uno de los planos perpendiculares al plano de lascado, pudiendo adoptar una forma recta (RE), cóncava (CN), convexa (CX), sinuosa (SI) o irregular (I).

Atributos.-Huellas características de cada tipo de fractura que son observables en su superficie: rebaba (RB), negativos y positivos (NP) y machacamientos, exfoliaciones o astillados (AS).

Causa.-Posible origen de la fractura: flexión (FL), percusión (PE), térmica (TE) o indeterminada (IN).

Determinadas hojas o lascas de sílex muy delgadas pueden ser fracturadas mediante torsión, dando lugar a un plano de fractura alabeado. No hemos reservado ningún campo a este tipo de causa por ser en general poco frecuente y no constatarse en nuestro conjunto.

Normalmente, se asocia un plano de fractura cóncavo-convexo con rebaba a una flexión; un plano ligeramente cóncavo o convexo, con huellas de negativo o positivo (contrabulbo, bulbo, ondas, etc.), respectivamente, a una percusión (otra pista son las posibles huellas de impacto que puedan observarse en alguna de las superficies que delimitan la fractura); y un plano irregular y exfoliado a una fractura térmica.

Los esfuerzos de flexión (véanse ejemplos en Lámina III, Fig. I) tienen lugar sobre las caras del soporte: uno o varios, "centrales", sobre una de las caras - normalmente el reverso, ya que, en dicho caso, la lasca ofrece menos resistencia a la rotura- $y$ otro/s a cada lado de los anteriores hacia los extremos, pero distantes de éstos y aplicados en sentido contrario.

Las flexiones se dan frecuentemente en soportes delgados y alargados o muy anchos, pues se requiere una cierta longitud de par (D) para que sean efectivos los pares de fuerza (por ejemplo, los pares $F_{1} F_{1}{ }^{\prime} D_{1}$ de la figura IA y $F_{2} F_{2}{ }^{\prime} D_{2}$ de la figura IB de la Lámina III) producidos por 
los esfuerzos que soporta la lasca. Como, por lo general, las lascas son más largas que anchas, encontraremos un alto porcentaje de fracturas por flexión distales o proximales.

La percusión, en cambio, puede tener lugar sobre una de las caras del soporte o sobre una parte de su contorno (lateral, frente, canto o talón).

Las rebabas producidas por flexión a veces abarcan transversalmente gran parte de la superficie de la fractura, confiriéndole un marcado carácter convexo. Los negativos de éstas, por el contrario, presentarán una superficie cóncava con una pequeña rebaba. Entre las fracturas por percusión se encontrarían las producidas a partir del punto de impacto durante la talla (p.e., las "diametrales") pero, también pueden producirse fracturas "pasivas" transversales u oblicuas al eje tecnológico. Éstas pueden presentar morfologias semejantes a las de flexión, bien porque la lasca se refleje o se quiebre en varios tramos al desprenderse del núcleo, sobre todo en la obtención de láminas largas y delgadas.

Las fracturas producidas mediante percusión sobre yunque suelen presentar astillamientos.

Cro.-Cronología. En este apartado constatamos aquellas fracturas que son recientes mediante sus correspondientes signos distintivos.

\section{BLOQUE VI $\rightarrow$ MAGNITUDES (Véase gráfico 5)}

Peso.-Se expresa en gramos. Es interesante conocer este dato en sí mismo para extraer conclusiones sobre la elección de la materia prima, su aprovechamiento, aptitud, etc. Igualmente se puede relacionar con otras caracteristicas como el volumen de la pieza.

Longitud.-Contemplamos en este apartado la longitud según el eje tecnológico (ET) y la longitud máxima real de la pieza (MAX), las cuales

\begin{tabular}{|c|c|c|c|c|c|c|c|c|c|c|c|c|c|c|c|c|c|c|}
\hline \multicolumn{19}{|c|}{ MAGNITUDES } \\
\hline \multirow{3}{*}{ PESO } & \multirow{2}{*}{\multicolumn{2}{|c|}{ LONOITUD }} & \multicolumn{8}{|c|}{ ANCHURA } & \multicolumn{8}{|c|}{ GROSOR } \\
\hline & & & \multicolumn{3}{|c|}{ maxima } & & \multicolumn{3}{|c|}{ OTRAS } & & \multicolumn{3}{|c|}{ maxima } & & \multicolumn{3}{|c|}{ OTRAS } & \\
\hline & ET & $\max$ & 0.25 & 0.50 & 0.75 & 1 & 0 & 0.50 & 0.75 & 1 & 0.25 & 0.50 & 0.75 & 1 & 0 & 0.50 & 0.75 & 1 \\
\hline 48 & 74 & 71 & 56.5 & & & & 35 & 44 & 32 & 旦 & 13,5 & & & & 13,5 & 95 & 8,5 & 0 \\
\hline 84 & 96.5 & 20.5 & & 53 & & & 45 & 40 & 20 & $B$ & & 21 & & & 15 & 20 & 15 & 3,5 \\
\hline 29 & & 55 & 43 & & & & & 44 & 33,5 & 25 & 15 & & & & & 12,5 & 12.5 & 0 \\
\hline 97 & & 96 & & 55 & & & 47 & 47 & 34 & & & 23,5 & & & 17.5 & 20 & 11 & \\
\hline
\end{tabular}

Gráfico 5: Bloque VI. 
(véase lámina III, Fig. II), normalmente, coinciden o difieren poco. La longitud del eje tecnológico no se medirá cuando haya una o más fracturas que le afecte. Sin embargo, la longitud máxima de la pieza se medirá en todos los casos, independientemente que sea la real o no.

Anchura.-Respecto a la anchura máxima (véase Lámina III, Fig. II), ésta se mide y se posiciona, rellenando la casilla correspondiente, en el tramo longitudinal donde se localice. Los tramos o intervalos definidos son: Un cuarto de la longitud de la pieza $(0,25 \mathrm{~L})$ a partir del talón, entre $1 / 4 \mathrm{~L}$ y la mitad de la pieza $(0,5 \mathrm{~L})$, de ésta a tres cuartos de la misma $(0,75 \mathrm{~L})$, y de $3 / 4 \mathrm{~L}$ al extremo distal $(L=1)$. En los casos excepcionales en que la anchura máxima se dé en dos o más tramos longitudinales, se rellenará más de una casilla. Por lo general, sólo habrá una anchura máxima, y por lo tanto solamente se cumplimentará una de las casillas.

En el subapartado otras anchuras se pondrá en la casilla correspondiente la anchura del talón ( $L=0)$, la obtenida en la mitad longitudinal de la pieza $(L=0,5)$, a tres cuartos del talón $(L=0,75)$ y en el extremo distal ( $L=1)$.

Cuando exista fractura no se medirá la magnitud o magnitudes afectadas por la misma. En el caso de fractura lateral de extremo proximal a extremo distal, no se tomará ninguna medida de anchura, pero si la pieza está fracturada proximal, distal, o proximal y distalmente, puede medirse la anchura a la mitad y a dos tercios de la longitud del fragmento, si tenemos algún atributo que nos permita orientar la pieza y si conviene a nuestros intereses, a sabiendas de que dichas medidas no son las correspondientes a esas longitudes en la lasca originaria.

Una pieza que tenga la casilla "O" en blanco y las demás con números, presentará fractura proximal. Cuando su anchura en el límite distal $(L=1)$ sea cero, acabará en punta, aunque ésta puede ser diédrica si presenta grosor, etc.

Grosor.-De manera análoga se rellenan los campos correspondientes al grosor, y de forma similar pueden analizarse la ausencia de magnitudes o sus valores. Así, por ejemplo, toda pieza con anchura en el límite distal (valor en « 1 \%), pero sin grosor en dicho extremo (valor 0 en “1»), presenta un filo transversal al eje. Cuando la anchura y el grosor en "1" es cero, la pieza tiene una punta aguzada.

BLOQUE VII $\rightarrow$ ATRIBUTOS (Véanse gráficos 6a y 6b)

Cada uno de los elementos morfotecnológicos derivados de las acciones de talla. 
Ficha analítica para el estudio morfo-tecnológico de la industria lítica no reelaborada

\begin{tabular}{|c|c|c|c|c|c|c|c|c|c|c|c|c|c|c|c|c|c|c|c|c|c|}
\hline & & & & & & & TRIBUTO & & & & & & & & & & & & & & \\
\hline SOP & DRTES & & & & ORT & & & & & $\mathrm{co}$ & WO & & & BULE & & & & & NOAS & & HUELLAS \\
\hline 1 & $P$ & & & GRADO & & DKAF & FECTADA & & A & $D$ & $N$ & $\mathbf{N}$ & A & \begin{tabular}{|l|}
0 \\
\end{tabular} & $\mathrm{~N}$ & $N$ & APRE & & DEST & $N$ & 1 МРАСTO \\
\hline & & & & 01810 & $M$ & TPL & LDURE & TA & & E & & & $P$ & E & $A$ & & DाM & & $D|A| F$ & A & $0: 11=$ \\
\hline 1 & & & 1 & & 1 & $1]$ & 1 & & 1 & & & & 1 & 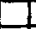 & & & & & & 1 & (1) \\
\hline 1 & & & 1 & & & 1 & ᄂ & 1 & & 1 & & & & 1 & & & & & & 1 & 1 \\
\hline 1 & & [ & & & & & & & & & & 1 & & . & & 1 & 111 & 1 & & & 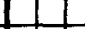 \\
\hline 1 & & & & & & 1 & & 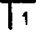 & & 1 & & & & 1 & & & \begin{tabular}{l|l|}
1 & 1 \\
\end{tabular} & 1 & & & 9 \\
\hline
\end{tabular}

Gráfico 6a: Bloque VII.

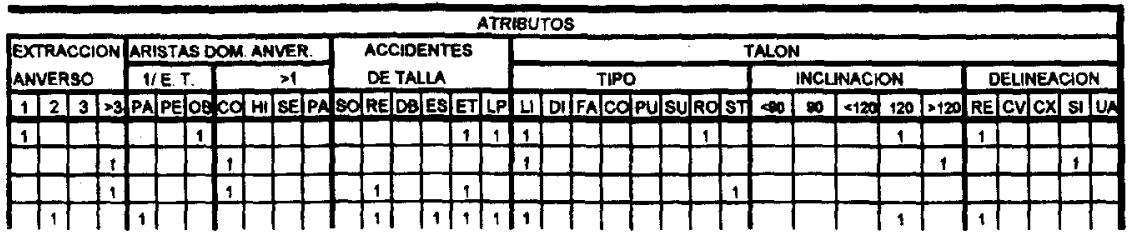

Gráfico 6b: Bloque VII.

Soportes.-Productos procedentes de los procesos de desbastado y acondicionamiento, lascado y retoque con caracteristicas morfotécnicas determinadas. Hemos considerado en nuestra ficha cuatro campos:

LA .- Lasca.

HO .- Hoja.

PA .- Producto de acondicionamiento (arista, flanco, tableta, etc.).

OT .- Otros (fragmento, chunk, debris, golpe de buril, ...).

Córtex.-Porción periférica no cristalina cuya estructura, textura y/o composición difiere del resto de la roca. En el caso de las rocas silíceas, las cuales por lo general se presentan en forma de nódulos o plaquetas, el córtex suele ser restos de roca-caja que se localizan en su contorno externo. Las cuarcitas son muy frecuentes en forma de cantos rodados, en cuyo caso, el córtex es la superficie externa de éstos, resultante de una alteración de los elementos superficiales de la roca.

Grado .- Porcentaje de roca-caja en anverso y talón. Se describen cinco campos: sin córtex $(0)$, menos del $30 \%(30)$ de la superficie espedificada, entre 30 y $60 \%$ (60), entre 60 y $<100 \%(100)$, y todo el anverso y talón con córtex (TO).

Zona afectada .- Donde se localiza longitudinal (D, M, P) y lateralmente (LD, LI) el córtex, y si éste alcanza al reverso (RE), lo que nos da una idea de su profundidad, o está presente en el talón (TA). 
Estos campos pueden suministrar, entre otra información, la posición del soporte en la cadena operativa.

En ocasiones es difícil discernir si se trata de restos de córtex o de pequeñas "islas" internas de roca-caja, que no han experimentado el proceso de disolución y sustitución por la sílice. Más raras veces, determinadas concreciones pueden también llevarnos a equivocos.

Cono.-Protuberancia, generalmente de forma cónica o esférica, que arranca del punto de impacto y es visible, parcialmente, en el extremo proximal de la cara de lascado.

Bulbo.-Convexidad que presenta la superficie de la cara de lascado desarrollada a partir de su extremo proximal.

Ondas.-Deformaciones lineales (inflexiones) de la superficie de lascado que aparentan tramos de circunferencias concéntricas, son perpendiculares al eje tecnológico y cóncavas respecto al extremo proximal.

Los tres atributos arriba definidos - los más característicos de las lascas-son consecuencia de la percusión o presión por las que son extraídas y del comportamiento elástico y amortiguador de la materia prima, especialmente de las rocas silíceas de grano fino. Así pues, dependen del impacto o fuerza recibida, tipo de materia prima, elasticidad del percutor, forma de prensión del núcleo y otros factores, como los tratamientos previos de la materia prima, que modifiquen sus propiedades físicas o químicas.

Estos atributos, por tanto, pueden ser indicativos, para una materia prima dada, de determinadas acciones de talla o, suponiendo pautas de talla similares, diferentes calidades de materia prima.

En el caso de extracciones mediante presión, percutor lítico de pequeño volumen o percutor elástico, estos atributos pueden ser imperceptibles a simple vista.

La ubicación del cono y del bulbo es conocida, por lo que se ha prescindido de parámetros zonales, constatándose únicamente si dichos atributos son apreciables (AP), destacados (DE), no apreciables (NA) o no conservados (NC).

Para las Ondas, sí hemos estimado oportuno registrar su posición a lo largo de la pieza (D, M, P) según su grado de desarrollo: Apreciables (APRE), destacadas (DEST), y no apreciables (NA). En su formación y en la intensidad con que se manifiestan intervienen múltiples factores, entre ellos la forma de prensión del núcleo, sobre todo cuando es una extracción controlada, que puede amortiguar la onda expansiva o, por el con- 
trario, reflejarla, acentuando la deformación del reverso. Las piezas reflejadas suelen presentar ondas distales destacadas.

Huellas de impacto.-Marca/s de la superficie del talón en el punto donde se ha aplicado una presión o percusión para la extracción de un soporte. Por lo general las huellas se caracterizan morfológicamente por uno o más círculos concéntricos y se localizan próximas al reverso y al cono. Cuando se observan más de una, se supone que ha habido uno o más golpes fallidos.

Extracción anverso.-Cada uno de los negativos, o fragmentos de éstos, originados por el lascado previo a la obtención del soporte, y que se conservan en él. Las extracciones a que nos referimos son de cierta magnitud, no se tienen en cuenta en este apartado las pequeñas descamaciones producidas en los diferentes retoques.

El contabilizar su número en anverso nos ayuda a hacernos una idea del grado de explotación y/o preparación del núcleo, así como nos aporta información sobre la morfología y complejidad de la lasca. Dicha información está asociada y complementada con la contenida en el apartado relativo a las aristas dominantes. En el caso concreto de una lasca kombewa (anverso y reverso, caras ventrales) o de una lasca de descortezado de primer orden, no se rellenaría ninguna de las casillas correspondientes a estos campos. Cuando se aprecien o se puedan discernir extracciones en el anverso posteriores a la obtención del soporte, se señalarán en el apartado destinado a observaciones.

Aristas dominantes. - Se entiende por arista la línea resultante de la confluencia de dos negativos de extracciones, o la línea de encuentro entre un negativo y el córtex originario de la superficie del núcleo presente en el anverso.

Una o dos extracciones dan lugar a una sola arista (1/ET), la cual podrá ser paralela (PA), perpendicular (PE) u oblicua (OB) al eje tecnológico, reportándonos información sobre la dirección de "lascado" con relación a la/s extracción/es anterior/es. Por lo general, las hojas o láminas suelen tener sus aristas paralelas al eje tecnológico.

Cuando hay más de una arista dominante éstas podrán ser convergentes (CO) o hiperboloides (HI), entrecruzarse aleatoriamente (SE) o ser paralelas (PA). Para esta evaluación debe hacerse una abstracción y contabilizar la tendencia general de las aristas dominantes, fruto de las grandes extracciones presentes en la lasca.

Accidentes de talla.-Fenómenos, por lo general no deseados, que pueden producirse durante la talla y que dejan huellas apreciables y características en la lasca. Hemos contemplado los siguientes (véase Lámina I): 


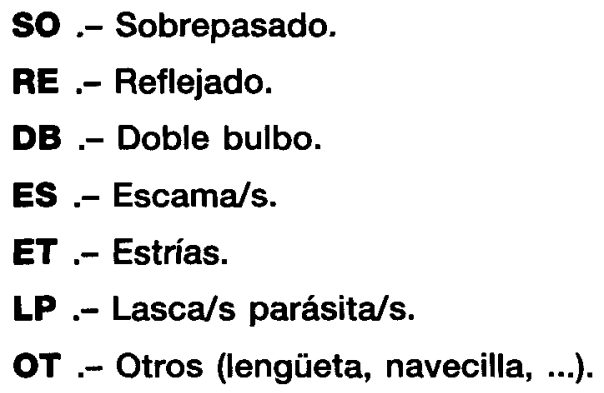

Talón.-Superficie del "plano» de percusión o de presión que se conserva en la lasca (véase Lámina II).

Tipo .- liso (LI), diedro (DI), facetado (FA), cortical (CO), puntiforme (PU), suprimido (SU), roto (RO), o sin talón (ST).

Inclinación .- Angulo formado por el plano del talón y el plano dominante o medio de la zona proximal del anverso (ángulo " $X ")$. Este parámetro nos informa u orienta sobre la inclinación del frente de lascado respecto al plano de percusión. En realidad el ángulo que consideramos y medimos es el ángulo complementario (180-x) (véase Lámina III, Fig. III). Para ello, podemos utilizar una escuadra y un cartabón, apoyando uno sobre el anverso y el otro sobre el talón — según convenga-, ya que los valores son aproximados.

No se ha considerado el "ángulo de lascado» que forman las superficies del talón y del reverso, que, con muchas reservas, puede orientarnos sobre la dirección de la fuerza aplicada, la elasticidad o masa del percutor...

Delineación .- En este caso entendemos por "delineación" la morfologia general que presenta el plano del talón respecto al observador: recto (RE), cóncavo (CV), convexo (CX), sinuoso (SI), y uniangular (UA).

\section{BLOQUE VIII $\rightarrow$ VARIOS}

Las piezas que presentan extracciones en el reverso implican una reelaboración del soporte y por lo tanto sobrepasan los objetivos de este modelo de ficha, mereciendo un estudio aparte. No obstante, se ha incluido un campo (EXRE) para constatar su existencia.

Igualmente, se ha incluido otro campo para señalar aquellas piezas que presenten retoque (RETO), con el fin de que éstas sean posteriormente estudiadas por separado para intentar asignarles una tipología. 
En el campo OBSERVACIONES se recogerán todas aquellas particularidades interesantes que no se contemplen en los demás campos.

Por último, queremos hacer especial mención a las muescas, cuyo estudio, en determinados casos, plantea problemas, ya que éstas pueden ser intencionadas (mediante retoque "clactoniense" y/o abrupto) 0 no. Estas, además, han podido ser aprovechadas como útil, produciéndose, en ocasiones, desconchados de uso que asemejan retoques. Por lo cual, en determinados conjuntos donde se presenten frecuentemente, seria interesante incluir en la ficha una serie de campos para su estudio pormenorizado, con independencia de su origen.

\section{RESUMEN}

En este trabajo se plantea un modelo de ficha para el análisis de industrias líticas no reelaboradas. Se trata de una sistematización de propuestas metodológicas que han venido apareciendo en los últimos años, fruto de una revisión crítica de las mismas y de nuevas aportaciones.

En la primera parte del artículo se explica la "filosofía» y la estructuración general del modelo propuesto. La ficha consta de varios bloques conceptuales con diferentes apartados y subapartados, que, en última instancia, pueden reducirse a cuatro categorias: grado, zona afectada, causa y tipo. Cada bloque tiene una estructura general semejante pero flexible, desarrollándose sus campos en mayor o menor cuantía en función de las caracteristicas de la industria objeto de estudio y de los intereses de la investigación.

En una segunda parte, se definen y explican los diferentes campos, valorándose críticamente su utilidad, y justificando su inclusión o no en cada caso.

Para la elaboración de este modelo se ha partido de una industria lítica de superficie, realizada básicamente sobre sílex, con diferente atribución cronocultural.

\section{ABSTRAC}

In this work a pattern file is proposed to analyse no-elaborated lithic industries. It consists of a systematization of methodological proposals which have lately been coming out. It is the result of a critical revision of the previously mentioned proposals, as well as new contributions. 
The first part of the article deals with the "philosophy" and general structuring of the proposed model. The file contains several conceptual blocks with different sections and "sub-sections", which can be divided into four categories: grade, affected area, cause and class. Each of these blocks has a general similar structure but flexible, to be developed its fields more or less depending on the features of every researched industry as well as the interests of the investigation.

In the second part of the article, the different fields of the file are explained and defined being criticaly valued its usefulness, and justified its inclusion o exclusion depending on every particular case.

The starting point to elaborate this pattem has been a lithic industry found at superficial level, made on flint with different crono-cultural attribution.

Nota.-Para aquellos términos no definidos, por supuestamente conocidos, o no del todo claros para el lector, remitimos a la consulta de la bibliografía que a continuación se adjunta.

\section{BIBLIOGRAFÍA}

BAENA, J., y Luque, M. (1990): "Modelo de análisis de industrias líticas no elaboradas", Xabiga núm. 6, págs. 44-58.

BeRNALDO de Quiros, F., et al. (1981): “Proyecto de análisis técnico para las industrias líticas", Trabajos de Prehistoria. Vol. 38, págs. 9-45.

Bustillo, M.A. (1976): «Estudio petrológico de las rocas silíceas miocenas de la cuenca del Tajo", Estudios Geológicos, núm. 32, págs. 451-497.

- (1978): “Significado de las rocas silíceas en la sedimentación miocena de la cuenca del Tajo", Estudios Geológicos, núm. 34, págs. 129-132.

- (1980): "Diagénesis de sílex", Revista del Instituto de Investigaciones Geológicas, vol. 34. Universidad de Barcelona, págs. 237-248.

Carbonell, E., et al. (1983b): «Elaboration d'un systéme d'analyse our l'étude des éclats bruts de débitage", Dialekiké, págs. 22-40.

InIZAN, M.L., et al. (1992): Préhistoire de la pierre taillée, III. Technology of knapped stone. Meudon (Francia), C.N.R.S., 127 págs.

LAPLACE, G. (1957): “Typologie analitique. Aplication d'une nouvelle méthode d'etude des formes et des structures aux industries à lames et lamelles", Quaternaria, t. 4, págs. 133-164.

- (1974): "De la dynamique de l'analyse structurale ou typologie analytique", Riv. Sc. Preist., Vol. XXIX.

MANSUR-FrANCHOMME, M.E. (1986): “Microscopia del material lítico prehistórico - Huellas de uso, alteraciones naturales, accidentales y tecnológicas-», Cahiers du Quaternaire, núm. 9. Bordeaux, C.N.R.S., pág. 286.

Merino, J.M." (1980): “Tipologia lítica», Munibe, Sup. núm. 4, 510 págs.

MorA, R., et al. (1991): «Un proyecto de análisis: El sistema lógico analítico (SLA), Treballs d'Arqueologia, I, págs. 173-199.

OWEN, L. (1982): "An analysis of experimental breaks on flint blades and flakes", Studia Praehistorica Belgica, 2, págs. 77-87.

ROCHE, H., y TIXIER, J. (1982): "Les accidents de taille", Studia Praehistorica Belgica, 2, págs. 65-76.

Semenov, S.A. (1981): Tecnología prehistórica. Madrid, Akal/Universitaria. 


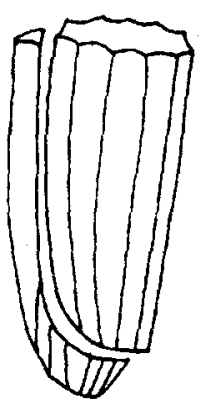

FIG. 1

SOBREPASADO

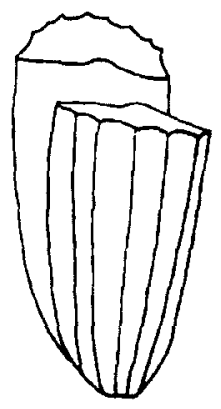

FKG. IV

FLANCO DE NUCLEO

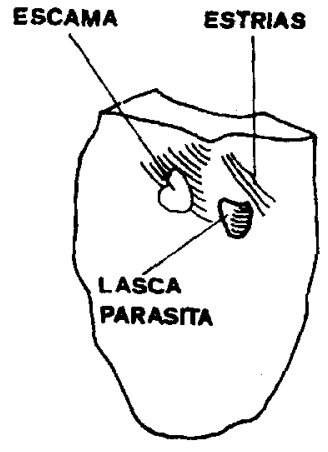

FIG. II

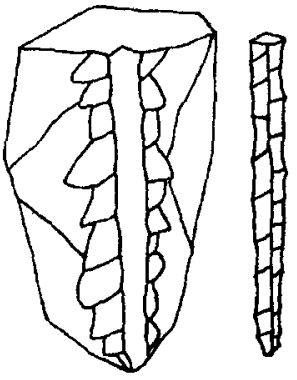

FIG. V ARISTA

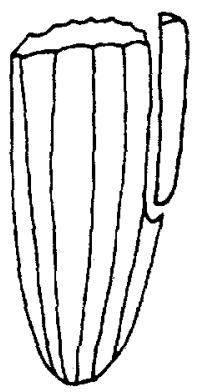

FIG. III REFLEJADO
FIG. $V$ I TABLETA DE NUCLEO

Lámina I: figuras. I, II, III. Principales accidentes de talla.

Figuras. IV, V, VI. Principales productos de acondicionamiento. 


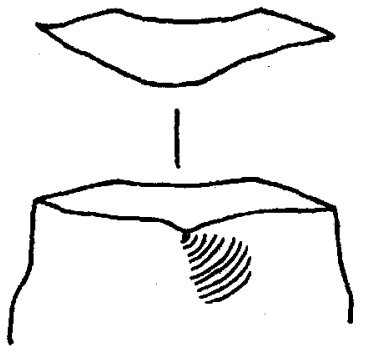

FIG.I TALON LISO

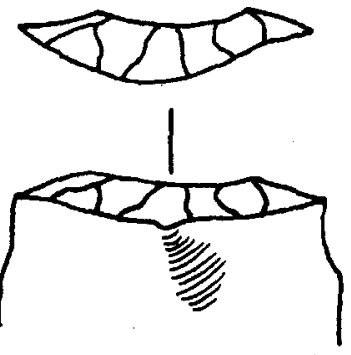

FG. III TALON FACETADO

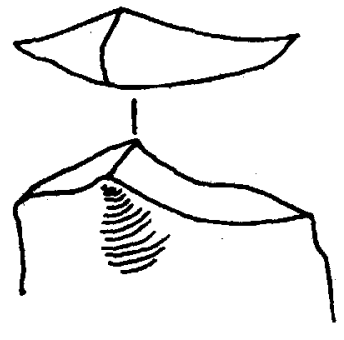

FIG. II TALON DIEDRO

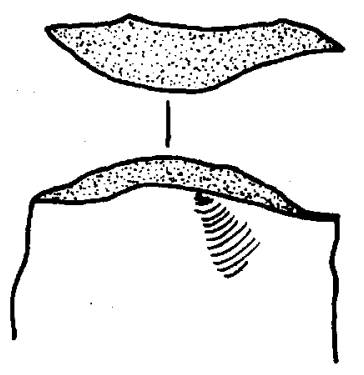

FIG. IV TALON CORTICAL

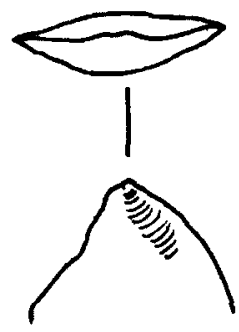

FIO. V TALON PUNTIFORME

Lámina II: Distintos tipos de talones. 

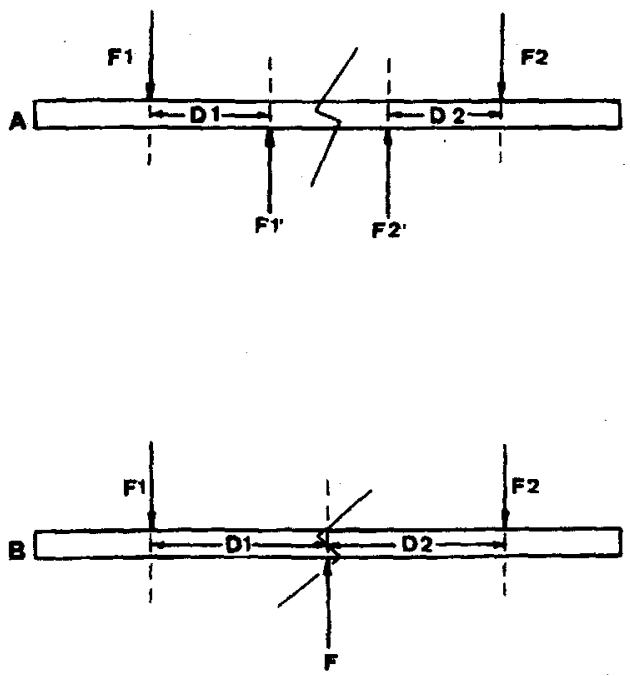

FIG. I

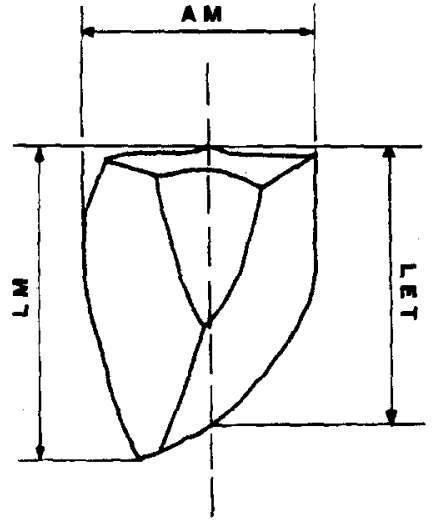

FiG. II

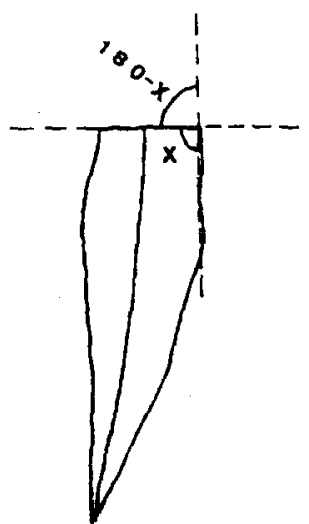

FG. III

Lámina III: fig. I. Representación esquemática de los esfuerzos que pueden tener lugar en las fracturas producidas por flexión;

fig. II. Principales medidas que se toman de un soporte litico;

fig. III. Croquis donde se explica como se mide la inclinación del plano de percusión con respecto al anverso del soporte. 